Case Report

\title{
Managing a Rivaroxaban Bleed: Understanding the Difficulties in Acute Reversal of the New Oral Anticoagulants through a Case Report
}

\author{
Naveen Nannapaneni, ${ }^{1}$ Robby Singh, ${ }^{1}$ Paulina Mckay, ${ }^{2}$ and Marwan Al-Hajeili ${ }^{3}$ \\ ${ }^{1}$ Department of Internal Medicine, Detroit Receiving Hospital/University Health Center, \\ Suite 2E, 4201 St. Antoine Street, Detroit, MI 48201, USA \\ ${ }^{2}$ Pharmacy Department, Detroit Medical Center, Detroit, MI, USA \\ ${ }^{3}$ Department of Internal Medicine, King Abdulaziz University, Jeddah, Saudi Arabia
}

Correspondence should be addressed to Naveen Nannapaneni; nnannapa@med.wayne.edu

Received 23 July 2014; Accepted 28 October 2014; Published 13 November 2014

Academic Editor: Kiyotaka Kawauchi

Copyright (C) 2014 Naveen Nannapaneni et al. This is an open access article distributed under the Creative Commons Attribution License, which permits unrestricted use, distribution, and reproduction in any medium, provided the original work is properly cited.

With the arrival of a new generation of oral anticoagulants significant burdens associated with warfarin's use on both the patient and the healthcare system have been alleviated. Nevertheless, a shortfall exists in regard to an agent or protocol for reversal of these new anticoagulants in the setting of an acute bleed. Our case of a patient presenting to the hospital with a vaginal bleed while on rivaroxaban highlights the difficulty in management without a clear protocol or agent for reversal of anticoagulation.

\section{Introduction}

For decades the mainstay of outpatient anticoagulation has been warfarin, an inhibitor of hepatic synthesis of vitamin $\mathrm{K}$ dependent coagulation factors [1]. Annual prescriptions for warfarin number in the tens of millions as it is used for a multitude of indications including treatment/prevention of venous thromboembolism and prophylaxis in nonvalvular atrial fibrillation and cardiac-valve replacement [1]. It requires close monitoring for its narrow therapeutic window, prolonged onset/offset, and variable response, all of which must be managed in the setting of several medication interactions, making it a problematic drug for both patient and physician [1]. With the arrival of a new generation of oral anticoagulants-direct factor Xa inhibitors rivaroxaban (Xarelto) and dabigatran (Pradaxa) and the direct thrombin inhibitor apixaban (Eliquis) - many of these burdens on both the patient and the healthcare system have been alleviated. These medications do not require close monitoring of anticoagulation parameters; they have fewer drug interactions through cytochrome enzymes, and no dietary restrictions of food, making them more convenient while still maintaining efficacy on par with, or better than, warfarin [2-4].
They have since gained FDA approval for additional indications [5] which will undoubtedly increase the number of users in the years to come. As more physicians begin to adopt these oral anticoagulants into their practice a concurrent increase in medication sequelae is to be expected, the most important of which is acute bleeding. There is no agent for immediate reversal of the new oral anticoagulants in the status quo [6] and the management of such cases can be difficult, as exhibited with our patient.

\section{Case Presentation}

A 39-year-old woman with a history of schizoaffective disorder and bilateral pulmonary emboli being treated with rivaroxaban presented to the Emergency Room (ER) with a two-week history of vaginal bleeding. She endorsed fatigue, dyspnea, and lightheadedness while associating abdominal cramping and low back pain with the bleeding. She reported no prior history of abnormal menstrual bleeding. She stated she was transitioned from warfarin to rivaroxaban approximately six weeks ago, with hospital records indicating a switch to rivaroxaban $20 \mathrm{mg}$ daily was made due to concerns 
TABLE 1: Timeline of hospitalization.

\begin{tabular}{lcccc}
\hline Hospital day no. & 1 & 2 & 3 & 4 \\
\hline Hgb (g/dL) & $6.8,8.3,7.2,5.7$ & $5.1,9,8.3$ & $9.3,9.7,9.2$ & $8.2,7.8,9.3$ \\
\hline PT (seconds) & 14.1 & 14.6 & 12.6 & 21.9 \\
\hline aPTT (seconds) & 27.2 & 21.5 & 1.1 & 9.5 \\
\hline INR & 1.3 & 1.3 & Endometrial ablation \\
\hline Intervention & $\begin{array}{c}1,800 \text { units of } \\
\text { prothrombin complex } \\
\text { intravenous fluids, } \\
\text { and 2 units of packed } \\
\text { red blood cells }\end{array}$ & $\begin{array}{c}2.5 \text { mg conjugated } \\
\text { estrogen and 2 units } \\
\text { of packed red blood } \\
\text { cells }\end{array}$ & $\begin{array}{c}\text { Balloon tamponade } \\
\text { and 2 units of packed } \\
\text { red blood cells }\end{array}$ & $\begin{array}{c}\text { End 1 unit of packed } \\
\text { red blood cells }\end{array}$ \\
\hline
\end{tabular}

Hgb: hemoglobin, PT: prothrombin time, aPTT: activated partial thromboplastin time, INR: international normalized ratio.

of noncompliance with warfarin monitoring. Physical exam revealed blood pressure $122 / 70 \mathrm{mmHg}$, heart rate 70 beats per minute, respiratory rate 18 breaths per minute with an oxygen saturation of $99 \%$ on room air, and a temperature of $36^{\circ} \mathrm{C}$. Pelvic exam showed fresh blood and clots in the vaginal vault, and after they were cleared, bleeding from a closed external cervical os was identified. Laboratory investigations showed her hemoglobin on presentation was $6.8 \mathrm{~g} / \mathrm{dL}, 4.0 \mathrm{~g} / \mathrm{dL}$ below routine lab work done one month before, platelet count $266,000 / \mu \mathrm{L}$, prothrombin time 14.1 seconds, activated partial thromboplastin time 27.2 seconds, and INR 1.3.

In the ER she was started on intravenous fluids and given 1,800 units of intravenous activated prothrombin complex concentrate (PCC) per ER protocol to attempt reversal of anticoagulation. Upon admission hematology was consulted and their recommendations of holding rivaroxaban, transfusing two units of packed red blood cells, and discontinuing the PCC therapy, due to the increased risk of thrombosis, were followed. Through the next day the patient continued to bleed with her hemoglobin dropping from $8.3 \mathrm{~g} / \mathrm{dL}$ after the initial transfusion to $5.7 \mathrm{~g} / \mathrm{dL}$ and attempts to stop the bleeding with $2.5 \mathrm{mg}$ intravenous conjugated estrogen recommended by gynecology were unsuccessful. By the third day of admission the patient had received an additional 4 units of packed red blood cells with improvement in the hemoglobin to $8.3 \mathrm{~g} / \mathrm{dL}$. Notably, repeat coagulation studies revealed a prothrombin time of 14.6 seconds, activated partial thromboplastin time of 21.5 seconds, and INR 1.3 (Table 1). Gynecology attempted balloon tamponade which was also unsuccessful in inducing termination of bleeding. After discussing the implication to her fertility, the patient was agreeable to a dilation and curettage with endometrial ablation the next day which resulted in cessation of bleeding and stabilization of hemoglobin to $9.5 \mathrm{~g} / \mathrm{dL}$ after one additional unit of packed red blood cells was transfused. She was discharged on fondaparinux with hematology follow-up.

\section{Discussion}

In summary, our patient with a history of unprovoked pulmonary emboli being treated with rivaroxaban presented with endometrial bleeding necessitating attempts at bleeding termination including unsuccessful mechanical compression and PCC, ultimately requiring endometrial ablation. The inciting cause for her endometrial bleed was likely an underlying anatomical abnormality that was provoked by the use of rivaroxaban as there are no reports of endometrial bleeding being a specific side effect of the medication. Further complicating her case was the concern of appropriate medication dosing as the patient provided varying reports to physicians as to the frequency she was taking the pills, informing some that she was taking it once daily and reporting to others that she was taking it twice a day, which could have led to supratherapeutic levels of anticoagulation.

The acute management of a bleed while on rivaroxaban is a challenge for any physician. In our case, the patient was given PCC based on the hospital's ER protocol. It was designed based on a study which reported efficacy of PCC to reverse the anticoagulant effect of rivaroxaban [7]. Since publication, this study has been met with some skepticism in regard to PCC being an antidote for reversal. First, it was conducted in nonbleeding healthy subjects and used endogenous thrombin potential and prothrombin times to gauge reversal, both of which are suboptimal methods of monitoring rivaroxaban [8]. The sensitivity of prothrombin time varies based on the reagent used and there is no standardized conversion to use across laboratories and institutions [8]. Moreover, it has been shown in animal models that an improvement in these coagulation studies did not always correlate with a reduction in clinical bleeding [9] and that the improvement observed in lab parameters was not always sustained [10]. Its availability and cost are additional prohibitive concerns limiting its widespread use [11].

Further relevant to our case, the use of PCC is also associated with an increased risk of thrombotic complications such as venous thromboembolism, disseminated intravascular coagulation, microvascular thrombosis, and myocardial infarction [12-14] which was a significant concern given our patient's history of underlying unprovoked pulmonary emboli. Without a workup for a potential underlying thrombophilia the use of PCC may have increased her risk for any of these ramifications. This concern was shared by the hematology consultants who felt the risk of additional clot burden 
for the patient did not justify continuing the PCC started in the ER. This risk of thrombosis needs to be addressed on a case-by-case basis depending on the indication for anticoagulation. For example, a different threshold for the clinician's concern may exist for a patient on anticoagulation for nonvalvular atrial fibrillation versus a patient with an underlying hypercoagulable state being treated for a venous thromboembolism. The use of PCC for a major bleed remains a topic for debate and at present it has not gained FDA approval as an agent for acute reversal.

In contrast, bleeding with warfarin is managed with a clear protocol for emergent reversal based on the INR as a result of extensive research into this complication [15]. Therapies ranging from holding doses of warfarin, vitamin K replacement, fresh frozen plasma (FFP), or PCC are used depending on the INR and clinical setting. Recent trials using current reversal modalities for rivaroxaban have not yielded promising results. Interventions such as FFP and recombinant activated factor VII have been studied and shown to be ineffective [16]. The quantity of clotting factors in FFP is relatively miniscule-one dose of PCC has the amount of clotting factors found in 8-16 units of FFP-and thawing and transfusing such large volumes would be impractical in an acute setting. As a result, current therapies entail supportive management including red blood cell transfusions and local mechanical compression for minor bleeds. Due to its shorter half life, 9-13 hours for rivaroxaban versus 2060 hours for warfarin, holding the medication will likely suffice for such cases. Surgical intervention based on cause and severity should also be considered, especially in major bleeds.

Attempts at developing a recombinant factor Xa, which would allow for immediate reversal by providing a competitive binding site for the $\mathrm{Xa}$ inhibitor and permit normal progression through the coagulation cascade, are in process and would likely provide an idyllic solution [17]. Until that time, additional investigation into the utility of PCC in actively bleeding patients on rivaroxaban is warranted. Its use must be thoroughly considered on a case-by-case basis to weigh the known risks and the potential benefits. With the ever increasing number of patients using these new oral anticoagulants the frequency of acute bleeds similar to our case encountered by physicians will continue to rise and developing a medication and protocol for immediate reversal is of paramount importance to prevent morbidity and mortality associated with new oral anticoagulant-induced bleeds.

\section{Conflict of Interests}

None of the authors have any conflict of interests to disclose.

\section{References}

[1] W. F. Peacock, M. M. Gearhart, and R. M. Mills, "Emergency management of bleeding associated with old and new oral anticoagulants," Clinical Cardiology, vol. 35, no. 12, pp. 730-737, 2012.
[2] A. Fawole, H. A. Daw, and M. A. Crowther, "Practical management of bleeding due to the anticoagulants dabigatran, rivaroxaban, and apixaban," Cleveland Clinic Journal of Medicine, vol. 80, no. 7, pp. 443-451, 2013.

[3] M. R. Patel, K. W. Mahaffey, J. Garg et al., "Rivaroxaban versus warfarin in nonvalvular atrial fibrillation," The New England Journal of Medicine, vol. 365, no. 10, pp. 883-891, 2011.

[4] S. J. Connolly, M. D. Ezekowitz, S. Yusuf et al., "Dabigatran versus warfarin in patients with atrial fibrillation," The New England Journal of Medicine, vol. 361, no. 12, pp. 1139-1151, 2009.

[5] J. L. Gordon, T. C. Fabian, M. D. Lee, and M. Dugdale, "Anticoagulant and antiplatelet medications encountered in emergency surgery patients: a review of reversal strategies," The Journal of Trauma and Acute Care Surgery, vol. 75, no. 3, pp. 475-486, 2013.

[6] N. I. Awad and C. Cocchio, "Activated prothrombin complex concentrates for the reversal of anticoagulant-associated coagulopathy," P and T, vol. 38, no. 11, pp. 696-701, 2013.

[7] E. S. Eerenberg, P. W. Kamphuisen, M. K. Sijpkens, J. C. Meijers, H. R. Buller, and M. Levi, "Reversal of rivaroxaban and dabigatran by prothrombin complex concentrate: a randomized, placebo-controlled, crossover study in healthy subjects," Circulation, vol. 124, no. 14, pp. 1573-1579, 2011.

[8] J. L. Thigpen and N. A. Limdi, "Reversal of oral anticoagulation," Pharmacotherapy, vol. 33, no. 11, pp. 1199-1213, 2013.

[9] A. Godier, A. Miclot, B. Le Bonniec et al., "Evaluation of prothrombin complex concentrate and recombinant activated factor VII to reverse rivaroxaban in a rabbit model," Anesthesiology, vol. 116, no. 1, pp. 94-102, 2012.

[10] M. Franchini and G. Lippi, "Prothrombin complex concentrates: an update," Blood Transfusion, vol. 8, no. 3, pp. 149-154, 2010.

[11] E. Perzborn, A. Gruber, H. Tinel et al., "Reversal of rivaroxaban anticoagulation by haemostatic agents in rats and primates," Thrombosis and Haemostasis, vol. 110, no. 1, pp. 162-172, 2013.

[12] E. Perzborn, S. Heitmeier, L. Volker, and A. Buchmuller, "Reversal of rivaroxaban-induced anticoagulation with prothrombin complex concentrate, activated prothrombin complex concentrate and recombinant activated factor VII in vitro," Thrombosis Research, vol. 133, no. 4, pp. 671-681, 2014.

[13] B. Sørensen, D. R. Spahn, P. Innerhofer, M. Spannagl, and R. Rossaint, "Clinical review: Prothrombin complex concentrates-evaluation of safety and thrombogenicity," Critical Care, vol. 15, no. 1, pp. 201-210, 2011.

[14] D. Nitzki-George, I. Wozniak, and J. A. Caprini, "Current state of knowledge on oral anticoagulant reversal using procoagulant factors," Annals of Pharmacotherapy, vol. 47, no. 6, pp. 841-855, 2013.

[15] J. Ansell, J. Hirsh, E. Hylek, A. Jacobson, M. Crowther, and G. Palareti, "Pharmacology and management of the vitamin $\mathrm{k}$ antagonists: american college of chest physicians evidencebased clinical practice guidelines," Chest, vol. 133, no. 6, pp. 160198, 2008.

[16] K. Babilonia and T. Trujillo, "The role of prothrombin complex concentrates in reversal of target specific anticoagulants," Thrombosis Journal, vol. 12, no. 1, article 8, 2014.

[17] G. Lu, F. R. Deguzman, S. J. Hollenbach et al., "A specific antidote for reversal of anticoagulation by direct and indirect inhibitors of coagulation factor Xa," Nature Medicine, vol. 19, no. 4, pp. 446-451, 2013. 


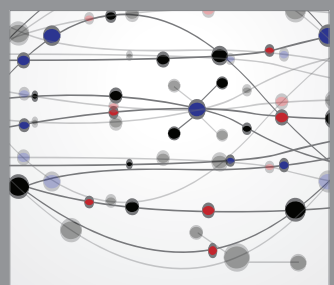

The Scientific World Journal
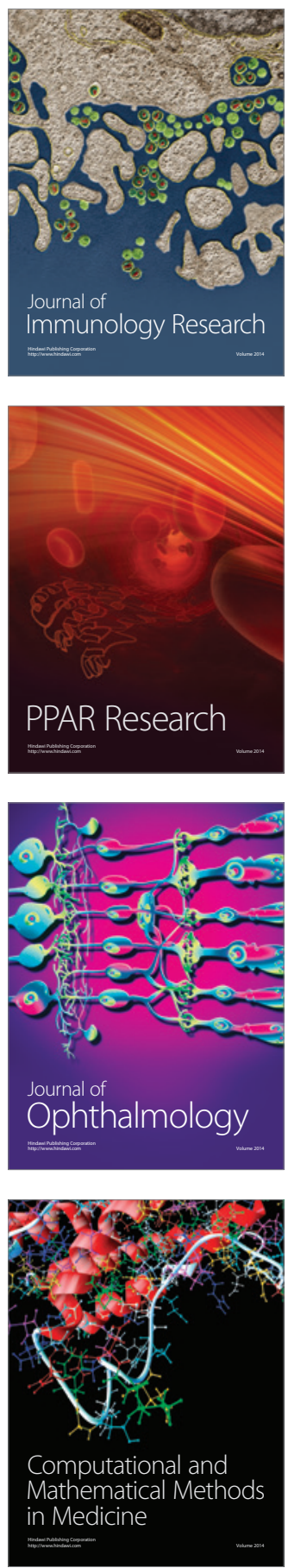

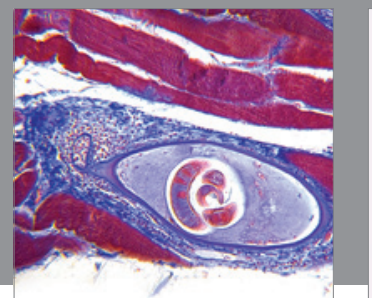

Gastroenterology

Research and Practice
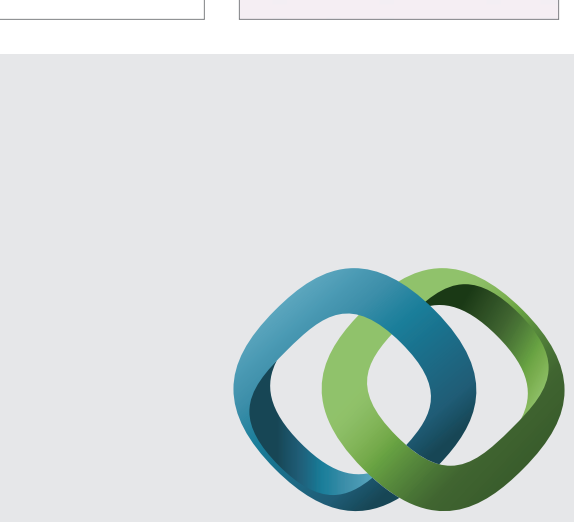

\section{Hindawi}

Submit your manuscripts at

http://www.hindawi.com
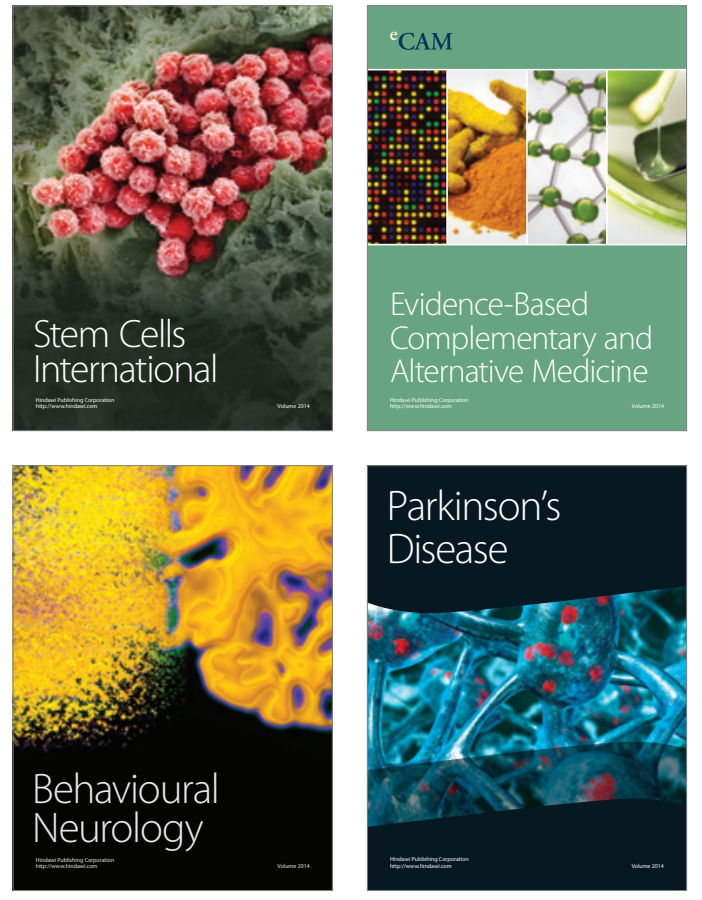
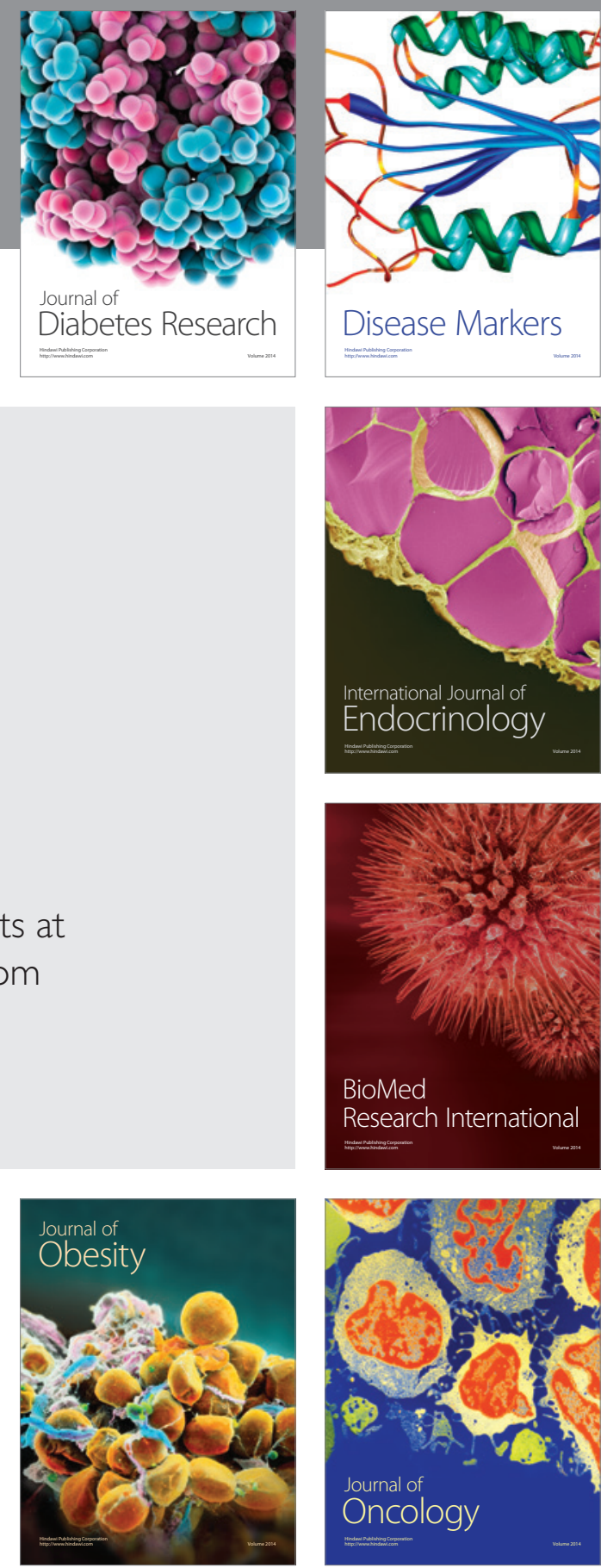

Disease Markers
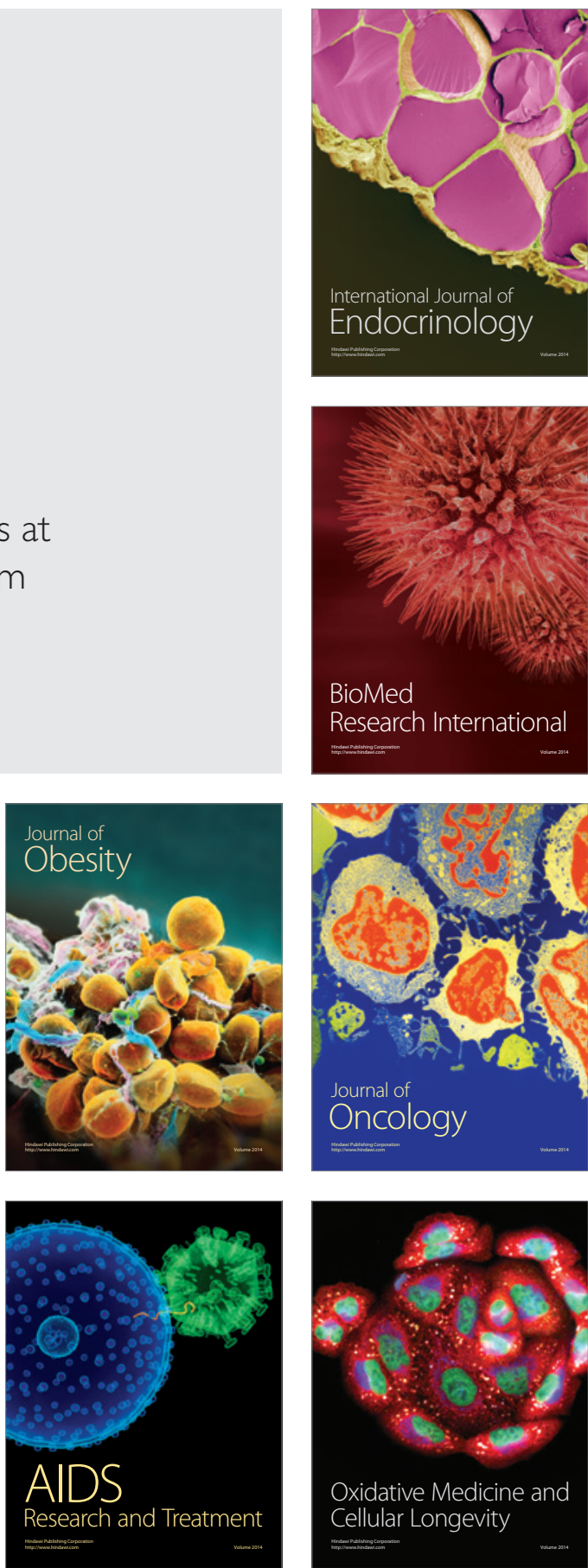